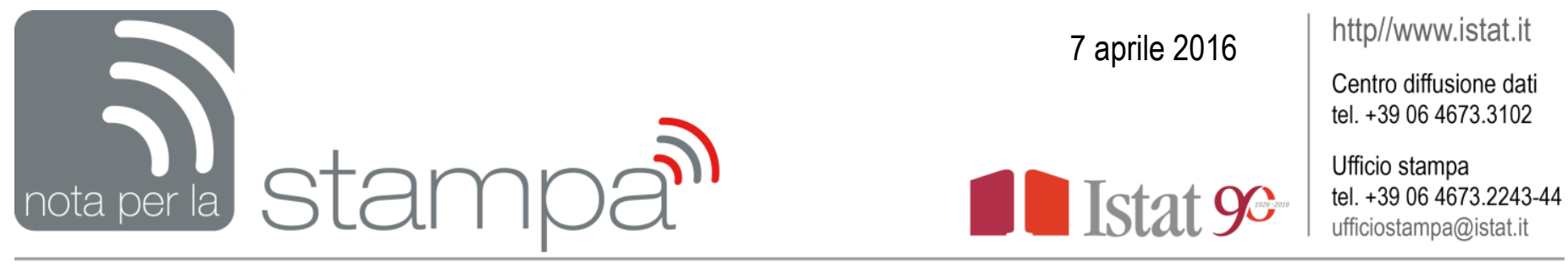

\title{
Noi Italia
}

\author{
Edizione 2016
}

Noi Italia offre una selezione di indicatori statistici che spaziano dall'economia alla cultura, al mercato del lavoro, alle condizioni economiche delle famiglie, alla finanza pubblica, all'ambiente, mettendo in luce le differenze regionali che caratterizzano il Paese e la sua collocazione nel contesto europeo.

Questa edizione è stata profondamente rinnovata per renderla più accessibile e più facile nella consultazione. Gli oltre 100 indicatori, ora distribuiti in sei macro aree e 19 settori, possono essere consultati in modo interattivo attraverso strumenti di visualizzazione grafica, con la possibilità di esportare i grafici sui propri siti internet. Per ciascun settore è possibile approfondire i diversi aspetti grazie alle Info disponibili e scaricare i dataset. È inoltre possibile acquisire l'intera base dati utilizzando l'icona Download dati presente nella homepage del sistema.

\section{POPOLAZIONE E SOCIETA'}

\section{Popolazione}

Nel 2014 I'Italia si conferma il quarto paese per importanza demografica dopo Germania, Francia e Regno Unito. Oltre un terzo della popolazione italiana è concentrata in tre regioni: Lombardia, Lazio e Campania. II Mezzogiorno è l'area più popolata del Paese anche se è cresciuta meno nel periodo 2004-2014.

Al $1^{\circ}$ gennaio 2015 ci sono 157,7 anziani ogni 100 giovani e 55,1 persone in età non lavorativa ogni 100 in età lavorativa, valori in continua ascesa negli ultimi anni.

Secondo le prime stime relative al 2015, per la prima volta negli ultimi 10 anni la speranza di vita alla nascita arretra, con un decremento di 0,2 punti per gli uomini $(80,1)$ e 0,3 per le donne $(84,7)$. Nel Mezzogiorno i valori della speranza di vita si confermano al di sotto della media nazionale.

Continua a diminuire il numero medio di figli per donna, nel 2014 si attesta a 1,37 mentre occorrerebbero circa 2,1 figli per garantire il ricambio generazionale. Se si considera l'età della madre, le regioni del Mezzogiorno si confermano, mediamente, quelle con le madri più giovani.

Con 3,2 matrimoni ogni mille abitanti, l'Italia rimane uno dei paesi dell'Ue28 in cui ci si sposa meno. Nel corso del 2014 in tutte le regioni si verifica una stasi o un calo (ad eccezione del Trentino-Alto Adige); il Mezzogiorno è la ripartizione con la nuzialità più alta, il Nord-ovest quella con meno matrimoni rispetto alla popolazione.

L'Italia presenta una bassa incidenza di divorzi, 8,6 ogni 10mila abitanti nel 2014; a livello europeo solo Irlanda e Malta registrano valori inferiori (anno 2013). Per le separazioni si sta verificando una convergenza tra le varie aree del Paese (14,8 e 14,6 ogni 10mila abitanti nel Centro-Nord e nel Mezzogiorno) mentre il divario Nord-Sud per i divorzi rimane ancora evidente (rispettivamente 9,8 e 6,6). 


\section{Stranieri}

All'inizio del 2015 risiedono in Italia oltre 5 milioni di cittadini stranieri (1,9\% in più rispetto all'anno precedente) che rappresentano l'8,2\% del totale dei residenti.

Alla stessa data sono regolarmente presenti 3.929.916 cittadini non comunitari (55mila in più rispetto al 2014). Il flusso in ingresso di cittadini non comunitari verso il nostro Paese risulta in flessione: nel corso del 2014 i nuovi permessi rilasciati sono stati quasi il 3\% in meno rispetto all'anno precedente. La riduzione dei nuovi ingressi ha riguardato soprattutto il Nord-est del Paese, mentre nel Mezzogiorno si è registrato un deciso aumento (quasi 8 mila in più), a seguito soprattutto degli arrivi per mare di persone in cerca di protezione internazionale.

II grado di istruzione degli stranieri è di poco inferiore a quello degli italiani; tra i 15-64enni quasi la metà degli stranieri ha al massimo la licenza media, il $40,1 \%$ ha un diploma di scuola superiore e il $10,1 \%$ una laurea (tra gli italiani il $15,5 \%$ ).

\section{Cultura e tempo libero}

Nel 2015 si stabilizza la quota di persone che leggono quotidiani $(47,1 \%)$ e aumenta leggermente quella di chi legge libri, anche se è ancora sotto il $50 \%$. Le percentuali maggiori di lettori si registrano fra i giovani e le donne. A livello territoriale tutte le regioni del Mezzogiorno presentano valori inferiori al dato nazionale ad eccezione della Sardegna.

Si conferma elevato, anche se in leggera diminuzione, l'utilizzo della Rete per la lettura di giornali, news o riviste; tra i giovani di 20-24 anni uno su due usa il web a questo scopo. Su scala europea I'Italia occupa l'ultima posizione insieme all'Irlanda (Anno 2014).

Nel 2015 l' $8,2 \%$ della popolazione di 6 anni e più legge online e scarica dal web libri e e-book, la quota sale a poco meno del $20 \%$ tra i giovani di 15-24 anni. Questa forma di fruizione culturale è più diffusa nel Nordovest, unica ripartizione dove si registra una crescita rispetto al 2014.

Cresce la partecipazione culturale. Negli ultimi anni sono aumentati i visitatori a musei/mostre e a siti archeologici/monumenti ma anche le persone che vanno al cinema (quasi il 50\% della popolazione). La fruizione di spettacoli o intrattenimenti fuori casa è piuttosto differenziata a livello territoriale: il divario tra ripartizioni è molto rilevante nel caso di visite a musei e monumenti, maggiori nel Centro-Nord.

Nel 2013 le famiglie italiane hanno destinato alla spesa per ricreazione e cultura mediamente il 6,5\% della spesa complessiva per consumi finali, una quota inferiore a quella riscontrata nel 2012. La riduzione accomuna tutte le ripartizioni; il valore più elevato di questa spesa si osserva nel Nord-ovest, mentre il Mezzogiorno presenta una quota inferiore alla media nazionale.

La propensione alla pratica sportiva è in crescita nel 2015 ma riguarda ancora solo un terzo della popolazione (più gli uomini che le donne); la quota più elevata si riscontra nel Nord-est $(39,4 \%)$, la più bassa nel Mezzogiorno (24,9\%). Poco meno di un quarto dei praticanti vi si dedica in modo continuativo.

\section{Criminalità e sicurezza}

Nel 2014 si conferma il trend in discesa per gli omicidi volontari $(0,78$ per 100 mila abitanti) e le rapine $(64,5)$. Viceversa risultano in aumento i furti denunciati, soprattutto i furti in appartamento $(420,9$ per 100 mila abitanti). Le differenze territoriali si sono in qualche caso attenuate. L'incidenza maggiore di omicidi continua a registrarsi in Calabria, la Campania si conferma la regione con il valore massimo di rapine, mentre il Centro-Nord presenta i tassi più elevati per i furti denunciati.

È di sesso femminile il $31,1 \%$ delle vittime di omicidio, nel $55 \%$ circa dei casi l'assassino è il partner o ex partner (dati 2014). A livello territoriale, l'Umbria e la Toscana presentano la percentuale più alta di vittime donne. Nel confronto con i paesi europei (per i quali sono disponibili i dati) l'Italia si conferma in una posizione intermedia per questa tipologia di omicidio.

Nel 2013 l'azione penale è iniziata per 1.029 persone ogni 100mila abitanti, mentre l'archiviazione ha interessato 1.058 persone. Le imputazioni sono state principalmente il furto e le lesioni personali volontarie. 
II sovraffollamento delle carceri è in netta diminuzione per il maggior ricorso a misure alternative alla detenzione (88,2 detenuti per 100mila abitanti nel 2014). In ambito europeo, nel 2013 I'Italia si colloca al di sotto della media europea e tra gli undici paesi con una presenza proporzionalmente minore di detenuti.

II rischio criminalità si conferma uno dei problemi maggiormente sentiti dai cittadini. Nel 2015 la quota di famiglie italiane che percepiscono un elevato rischio di criminalità nella zona in cui vivono sale significativamente $(41,1 \%$ dal $30,0 \%$ del 2014$)$, riprendendo il trend di crescita interrotto l'anno precedente.

\section{Condizioni economiche delle famiglie}

Nel 2015 la quota di persone soddisfatte per la propria situazione economica risulta in aumento $(47,4 \%)$ per il secondo anno consecutivo; a crescere sono soprattutto coloro che si dichiarano "abbastanza soddisfatti". II livello di soddisfazione per la situazione economica presenta una variabilità a livello territoriale: Mezzogiorno e Centro sono le ripartizioni in cui l'aumento delle quota rispetto al 2014 è più consistente (circa 4,5 punti percentuali in più).

Tra il 2013 e il 2014 l'incidenza della povertà - relativa e assoluta - è risultata sostanzialmente stabile. La povertà relativa coinvolge circa un decimo delle famiglie residenti, quella assoluta il 5,7\%.

Nel 2013 le famiglie residenti in Italia hanno percepito un reddito disponibile netto (esclusi i fitti imputati) pari, in media, a 29.473 euro, circa 2.456 euro al mese. II valore varia sensibilmente sul territorio: la provincia autonoma di Bolzano registra il livello più elevato, 17.500 euro in più rispetto al Molise, la regione con il valore più basso.

I paesi dell'Ue28 presentano notevoli differenze in termini di diseguaglianza. Nella graduatoria in ordine decrescente, l'indice di concentrazione colloca l'Italia al decimo posto $(0,324)$, con un valore poco più elevato di quello medio europeo $(0,309)$. Lungo la Penisola è la Sicilia a mostrare la concentrazione dei redditi più alta, con un indice pari a 0,365 , mentre II reddito è più equamente distribuito in Valle d'Aosta $e$ in Friuli-Venezia Giulia.

Nel 2014 l'indicatore di grave deprivazione materiale segna una riduzione, interessa l'11,6\% delle persone (12,3\% nel 2013). II valore del Mezzogiorno (19,9\%, oltre 4 milioni di individui), per quanto in forte diminuzione, è più elevato di quello rilevato in tutto il Centro-Nord $(7,2 \%$, quasi 3 milioni di individui).

\section{ISTRUZIONE E LAVORO}

\section{Istruzione}

Nel 2013 la spesa pubblica in istruzione incide sul Pil per il 3,6\% a livello nazionale, raggiunge il 6,0\% nel Mezzogiorno - dove è più numerosa la popolazione in età scolare - e scende al 2,7\% al Nord-ovest, unica ripartizione in cui l'incidenza della spesa è leggermente aumentata nell'ultimo anno.

Prosegue il miglioramento del livello di istruzione degli adulti. La quota di 25-64enni che hanno conseguito al massimo la licenza media è scesa dal $51,8 \%$ del 2004 al $40,5 \%$ del 2015 ma sfiora il $50 \%$ nel Mezzogiorno (48,8\%). Oltre la metà dei giovani 15-24enni è impegnata in un percorso di formazione superiore; i tassi di partecipazione sono più alti nel Centro-Nord, in particolare in Emilia-Romagna e Lazio.

Nel 2014 la quota di giovani che abbandonano precocemente gli studi in Italia è scesa al $15 \%(17,7 \%$ tra gli uomini e 12,2\% tra le donne), superando l'obiettivo nazionale del 16\% fissato dalla Strategia Europa 2020.

Sono oltre 2,3 milioni $(25,7 \%$ del totale) i giovani $15-29$ enni che nel 2015 non sono inseriti in un percorso scolastico e/o formativo e non sono impegnati in un'attività lavorativa. L'incidenza è più elevata tra le donne e nel Mezzogiorno.

Fra i $30-34$ enni, il $25,3 \%$ ha conseguito un titolo di studio universitario nel 2015 , un valore che si avvicina molto al $26 \%$ stabilito dalla stessa Strategia europea come obiettivo per l'Italia. 
L'aggiornamento durante l'arco della vita, fattore decisivo per l'integrazione nel mercato del lavoro, interessa nel 2014 l'8,0\% degli italiani tra i 25 e i 64 anni, valore in aumento ma ancora sotto la media europea $(10,7)$.

\section{Mercato del lavoro}

Nel 2015 risultano occupate oltre 6 persone in età 20-64 anni su 10, ma è forte lo squilibrio di genere a sfavore delle donne $(70,6 \%$ gli uomini occupati, $50,6 \%$ le donne) come il divario territoriale tra Centro-Nord e Mezzogiorno. Nella graduatoria europea relativa al 2014, solamente Grecia, Croazia e Spagna presentano tassi di occupazione inferiori a quello italiano mentre la Svezia registra il valore più elevato (74\%).

Sale al $14 \%$ l'incidenza del lavoro a termine nel 2015 , più alta nelle regioni meridionali $(18,4 \%)$ rispetto al Centro-Nord (12,5\%). Cresce di poco la quota di occupati a tempo parziale (18,5\%), con una distribuzione piuttosto uniforme sul territorio nazionale. In Europa, questa modalità di occupazione è diffusa soprattutto nei paesi nordici (50,3\% l'incidenza nei Paesi Bassi nel 2014), mentre lo è poco nei paesi dell'Est di più recente adesione all'Unione.

Il tasso di disoccupazione scende di 0,8 punti rispetto al 2014, riportandosi dopo due anni sotto il 12\%. La riduzione interessa entrambe le componenti di genere, ma risulta più rilevante per le donne. Rimangono forti le differenze territoriali, con un tasso nel Mezzogiorno di poco inferiore al $20 \%$.

Nel 2015 il tasso di disoccupazione dei giovani 15-24enni scende al 40,3\%, 2,4 punti percentuali in meno rispetto a un anno prima. II livello massimo si registra nel Mezzogiorno $(54,1 \%)$, soprattutto in Calabria, dove arriva al $65,1 \%$ e fra le ragazze $(58,1 \%)$.

Poco meno di sei disoccupati su dieci $(58,1 \%)$ cercano lavoro da oltre un anno, in riduzione dal $60,7 \%$ del 2014. Il calo della disoccupazione di lunga durata interessa oltre la metà delle regioni e ha coinvolto soprattutto le donne.

II tasso di mancata partecipazione, che tiene conto di quanti sono disponibili a lavorare pur non cercando attivamente lavoro, si attesta al 22,5\% nel 2015, in rallentamento di 0,4 punti sul 2014. La riduzione è leggermente maggiore nel Mezzogiorno, anche se in questa ripartizione il valore rimane più che doppio rispetto al Centro-Nord.

\section{SALUTE E WELFARE}

\section{Sanità e salute}

Nel 2013 la spesa sanitaria pubblica italiana si attesta intorno ai 2.400 dollari pro capite a fronte degli oltre 3.000 spesi in Francia e Germania. La quota di spesa sanitaria privata è pari al $22,6 \%$ del totale, inferiore di oltre un punto percentuale rispetto a quella tedesca ma superiore a quella francese.

I tumori e le malattie del sistema circolatorio sono le patologie per cui è più frequente il ricovero ospedaliero; tuttavia il ricorso al ricovero è in progressiva riduzione perché sempre più spesso le persone vengono curate in contesti assistenziali diversi dagli ospedali (day hospital o ambulatori). Nel 2013 i ricoveri per le malattie circolatorie e i tumori si confermano più elevati nelle regioni del Centro, rispettivamente $2.044,6$ e $1.212,6$ per 100mila abitanti.

In Italia la mortalità per tumori e malattie del sistema circolatorio è inferiore alla media europea; nel $2012 \mathrm{i}$ decessi per queste cause sono stati rispettivamente 27,0 e 34,4 ogni 10mila abitanti. Nel Mezzogiorno la mortalità per tumori è inferiore alla media nazionale, mentre è più elevata quella per malattie del sistema circolatorio.

Il tasso di mortalità infantile, importante indicatore del livello di sviluppo e benessere di un paese, continua a diminuire; nel 2013 in Italia è di 2,9 per mille nati vivi, tra i valori più bassi in Europa. 
Gli stili di vita degli italiani continuano al migliorare. Nel 2014 si riducono i consumatori di alcol a rischio $(15,5 \%)$, i fumatori $(19,5 \%)$ e le persone obese $(10,2 \%)$. A livello territoriale la quota più alta di consumatori di alcol si ritrova nel Centro-Nord mentre l'obesità è più diffusa nel Mezzogiorno.

\section{Protezione sociale}

In Italia la spesa per la protezione sociale nel 2013 è circa il 30\% del Pil mentre il suo ammontare per abitante sfiora gli 8mila euro l'anno. All'interno della Ue28, I'Italia presenta valori appena superiori alla media, sia in termini pro capite sia di quota sul Pil. In quest'ultimo confronto, i valori più elevati si registrano in Francia $(33,7 \%)$, quelli più contenuti in Lettonia $(14,4 \%)$.

La spesa per prestazioni sociali (19,5\% del Pil nel 2013; 5.208 euro pro capite) è solo in parte coperta dai contributi sociali $(14,2 \%$ del Pil) come emerge dall'indice di copertura previdenziale inferiore a 100 e in progressiva diminuzione dal 2008. Rispetto al 2012 è aumentata, invece, l'incidenza sul Pil della spesa per le pensioni $(16,9 \%)$.

\section{INDUSTRIA E SERVIZI}

\section{Turismo}

Nel 2014 si contano in Italia 158.412 esercizi ricettivi con più di 4,8 milioni di posti letto, in crescita rispettivamente dello $0,6 \%$ e del $2,6 \%$ sul 2013. L'offerta italiana è superiore a quelle di Germania, Spagna e Francia, ma inferiore, tra le altre, all'offerta di Croazia, Austria e Grecia.

Nel complesso degli esercizi ricettivi gli arrivi sono stati 106,5 milioni e quasi 378 milioni le presenze. La durata media del soggiorno nelle strutture è di 3,55 notti, in lieve calo rispetto al 2013. Tutte le regioni del Nord-est si collocano al di sopra della media nazionale per numero medio di notti trascorse dai clienti negli esercizi ricettivi, mentre le regioni del Nord-ovest risultano al di sotto. Nel Centro solo le Marche registrano un numero medio di notti $(5,02)$ superiore alla media nazionale

\section{Strutture produttive}

La crisi economica si è riflessa in un calo del numero delle imprese. Nel 2013, in Italia operano circa 62 imprese ogni mille abitanti, un valore tra i più elevati del continente europeo. Solo cinque paesi (Repubblica Ceca, Portogallo, Slovacchia, Svezia e Grecia) hanno una densità di imprese più elevata, mentre i principali partner (Francia, Germania e Spagna) presentano valori più bassi.

La dimensione media delle imprese italiane, pari a 3,8 addetti, è di gran lunga inferiore al dato medio europeo $(5,9)$. Sotto il profilo territoriale, la dimensione media si conferma più bassa nel Mezzogiorno $(2,8)$.

Il tasso di imprenditorialità - calcolato come rapporto tra numero di lavoratori indipendenti e totale dei lavoratori delle imprese - si attesta al 30,2\% e risulta secondo solo a quello della Grecia fra i paesi dell'Unione europea; tra le maggiori economie dell'area, Germania e Francia presentano quote decisamente più contenute $(8,3$ e 9,5\%). Sul territorio nazionale, la propensione all'imprenditorialità è maggiore nel Mezzogiorno (38\%) che nel Centro-Nord (28,2\%).

II turnover lordo delle imprese, che fornisce una misura del grado di dinamicità di un sistema economico, in Italia cresce per il terzo anno consecutivo (15,6\% nel 2013). I valori sono alquanto diversificati sul territorio: nel Mezzogiorno la popolazione di imprese risulta più instabile, mentre il Nord-est si caratterizza per una minore nati-mortalità delle imprese.

La struttura produttiva italiana presenta le peculiarità di alcune economie dell'area mediterranea, dove prevalgono le forme più legate alle tipicità del territorio. La micro impresa nel settore dei servizi domina il panorama delle attività del sistema economico italiano. Rispetto alla media nazionale, nel Mezzogiorno prevalgono le micro imprese, sia di servizi sia dell'industria, nel Nord-ovest è più diffusa la grande industria, nel Nord-est le micro e piccole imprese dell'industria e nel Centro le grandi imprese dei servizi. 
Dopo il recupero del biennio 2010-2011, nel 2013 prosegue la perdita di competitività delle imprese italiane, con 122,9 euro di valore aggiunto ogni 100 euro di costo del lavoro (124,6 nel 2012). Le regioni del Nordovest mostrano i livelli di competitività più elevati, mentre il Mezzogiorno registra valori inferiori alla media nazionale.

\section{Infrastrutture e trasporti}

Nel 2014 la rete autostradale italiana si estende per $6.844 \mathrm{~km}$ e rappresenta poco più del $9 \%$ di quella europea; L'Italia è tra i paesi dell'Unione a più bassa intensità autostradale, ben lontana dai valori di Spagna, Francia e Germania.

Nel 2014 il trasporto di merci su strada ha sviluppato un traffico di poco inferiore a 118 miliardi di tonnellate$\mathrm{km}$ (Tkm), in calo del 7,4\% rispetto al 2013. II volume di traffico italiano, pari a 19,4 milioni di Tkm per 10 mila abitanti, è inferiore a quelli di tutti i principali partner dell'area dell'euro e si pone tra i più bassi nell'Ue28.

Nel 2014 l'Italia dispone di una rete ferroviaria pari a 27,4 km ogni 100mila abitanti, con una disponibilità sostanzialmente analoga nel Centro-Nord e nel Mezzogiorno. In ambito europeo, il nostro è tra i paesi con estensione relativa minore, seguito da Regno Unito, Portogallo, Grecia e Paesi Bassi.

Il tasso di motorizzazione è pari a quasi 610 autovetture ogni mille abitanti, in lieve aumento rispetto al 2013. II Centro si conferma la ripartizione con il valore più elevato. Nel confronto europeo l'Italia è di gran lunga uno dei paesi più motorizzati, preceduta solo da Lussemburgo e Lituania.

Nel 2014 si è attenuato il trend discendente della mortalità per incidenti e le vittime della strada risultano 55,6 ogni milione di abitanti. La mortalità stradale presenta differenze territoriali significative, con sette regioni che registrano tassi inferiori alla media nazionale. In Italia il numero dei decessi per incidente stradale si conferma superiore a quello medio europeo e in confronto ai principali partner.

Malta e Cipro sono i primi paesi nell'Ue per traffico aereo di passeggeri in rapporto alla popolazione nel 2014, mentre il nostro Paese è tra quelli ad intensità medio-bassa. Lazio e Lombardia rappresentano più della metà del trasporto passeggeri sui voli aerei in Italia.

Nel 2015 l'87,3\% degli occupati e il 74,1\% degli studenti utilizzano un mezzo di trasporto per recarsi al luogo di lavoro o studio, privilegiando l'automobile. Nel Mezzogiorno c'è una maggiore propensione a spostarsi a piedi, mentre nel Nord-est è più frequente l'uso dei mezzi di trasporto, sia per gli occupati sia per gli studenti.

\section{Scienza, tecnologia e innovazione}

Nel 2013 la spesa per ricerca e sviluppo aumenta in Italia sia in termini assoluti sia in rapporto al Pil $(1,31 \%)$. Tale valore è inferiore a quello medio europeo $(2,03 \%)$, ancora distante dall'obiettivo nazionale della Strategia Europa 2020 (1,53\%) e dal target europeo del 3\%.

II 93,5\% delle imprese italiane con almeno 10 addetti si connette a Internet tramite la banda larga (anno 2014), un valore superiore alla media Ue28 ma ancora distante da quelli dei paesi europei di testa come Finlandia, Danimarca, Paesi Bassi e Slovenia (98\%). A livello regionale le imprese attive in Calabria e Basilicata sono in maggiore ritardo rispetto alla media, quelle del Nord-ovest appaiono le più performanti.

Gli addetti alla ricerca e sviluppo (in unità equivalenti a tempo pieno) nel 2013 sono 4,1 ogni mille abitanti, meno rispetto alla media europea $(5,4)$ e ai valori delle economie più importanti. Sul territorio nazionale, si confermano forti disparità e resta elevato il ritardo del Mezzogiorno.

Nel 2013 i laureati in discipline tecnico-scientifiche sono 13,5 ogni mille residenti tra i 20 e i 29 anni, valore inferiore alla media europea $(17,1)$. In ambito nazionale, il distacco del Mezzogiorno rimane forte $(-4,4$ punti rispetto al dato nazionale).

Nel 2015 il 60,2\% della popolazione italiana di 6 anni e più utilizza Internet e il 40,3\% si connette quotidianamente. La totalità delle regioni del Centro-Nord ha livelli di uso di Internet superiori al valore 
nazionale, nel Mezzogiorno la quota è invece più bassa. La posizione nazionale è decisamente inferiore alla media Ue28 (75\% nel 2014), solo Bulgaria e Romania registrano una quota più contenuta.

Poco più di sei famiglie su dieci si connettono a Internet tramite la banda larga; il Mezzogiorno, e in particolare la Calabria $(56,6 \%)$, si trovano in posizione svantaggiata. Nel confronto europeo, la quota di famiglie italiane è inferiore alla media dei 28 paesi $(78 \%$ nel 2014), mentre i valori più elevati si registrano nel nord Europa.

\section{AMBIENTE E AGRICOLTURA}

\section{Territorio}

L'Italia si conferma tra i paesi più densamente popolati dell'Unione europea: nel 2014 la densità della popolazione è di 201,2 abitanti per $\mathrm{Km}^{2}$, con un aumento di quasi 10 abitanti negli ultimi 10 anni.

Nel 2015 le aree protette comprese nella Rete Natura 2000 coprono il 19,3\% della superficie nazionale, collocando I'Italia al di sopra della media Ue28 (18,1\%). Oltre un quinto del territorio del Mezzogiorno è compreso in questa rete, Sicilia e Sardegna presentano i valori regionali più alti.

Gli indicatori sui permessi di costruire registrano nel 2013 un nuovo calo: ogni mille famiglie sono stati autorizzati circa $177 \mathrm{~m}^{2}$ di superficie utile abitabile (868 nel 2005). A livello regionale la situazione si presenta tuttora eterogenea, con valori molto inferiori a quelli medi in Toscana e Liguria e più alti nella provincia autonoma di Bolzano.

\section{Ambiente}

Nel 2014 continua il calo nella produzione di rifiuti urbani, 487,8 $\mathrm{Kg}$ per abitante, circa $3 \mathrm{~kg}$ in meno rispetto all'anno precedente. A livello territoriale, le maggiori quantità di rifiuti urbani si raccolgono nelle regioni del Centro Italia.

Prosegue la riduzione di rifiuti raccolti e smaltiti in discarica, nel 2014 sono 153,5 kg per abitante, 27,7 in meno rispetto al 2013. Le migliori performance si registrano per la provincia autonoma di Bolzano, la Lombardia e il Friuli-Venezia Giulia.

La raccolta differenziata, fattore strategico per la corretta gestione dei rifiuti, nel 2014 ha raggiunto l'obiettivo del $45 \%$ previsto dalla normativa nazionale per il 2008. In vetta alla graduatoria si collocano la provincia autonoma di Trento e il Veneto, dove si supera il 65\%, obiettivo previsto per il 2012. La Sicilia è invece la più lontana dai target (12,5\%).

Nel 2015 aumentano rispetto all'anno precedente le percentuali di famiglie che percepiscono inquinata l'aria della zona in cui vivono (36,7\%) e che evidenziano la presenza di odori sgradevoli $(20,5 \%)$. Sull'inquinamento dell'aria sono le famiglie del Nord-ovest a segnalare maggiormente il problema mentre la percezione di odori sgradevoli interessa di più quelle del Mezzogiorno.

\section{Agricoltura}

Nel 2013 il valore aggiunto per addetto del settore è pari a 142,8 euro per 100 euro di costo unitario del lavoro, confermando il trend di crescita. La migliore performance si riscontra nel Nord-ovest, grazie alla maggiore presenza di aziende di grande dimensione. All'interno delle ripartizioni geografiche, al Nord il livello più elevato dell'indicatore si registra in Lombardia, al Centro nel Lazio mentre tra le regioni meridionali si distinguono Calabria, Sicilia e Sardegna.

Nel 2014 la distribuzione di fertilizzanti semplici per uso agricolo si mantiene stabile $(0,1$ tonnellate per ettaro di SAU), mentre aumenta quella di principi attivi per ettaro di superficie agricola utilizzata (4,9 $\mathrm{Kg}$ per ettaro di SAU). 
I prodotti agroalimentari di qualità si confermano una componente significativa del comparto agroalimentare italiano, infatti il nostro Paese registra il numero di certificazioni più elevato a livello comunitario, 269 nel 2014. Oltre all'Italia le produzioni di qualità sono valorizzate in Francia, Spagna e Portogallo.

L'agriturismo conferma la tendenza strutturale alla crescita: tra il 2004 e il 2014 le aziende agrituristiche sono aumentate del $55,1 \%$, quasi il $36 \%$ è gestito da donne.

\section{Energia}

Nel 2014 diminuiscono sia i consumi elettrici (-3\% circa rispetto al 2013) che la produzione lorda di energia elettrica (-4,3\% rispetto al 2013). I consumi sono stati pari a $4.710,3 \mathrm{kWh}$ per abitante, il valore più basso degli ultimi 12 anni; la produzione lorda di energia elettrica si è invece attestata a 46 GWh per 10 mila abitanti, in flessione per il terzo anno consecutivo.

Per il nostro Paese entrambi gli indicatori energetici risultano inferiori alla media europea e a quelli degli altri paesi di grandi dimensioni. A livello territoriale, la produzione lorda di energia elettrica in rapporto alla popolazione si attesta su valori superiori alla media nazionale nelle ripartizioni settentrionali e nel Mezzogiorno. Nel 2014 i consumi elettrici per abitante risultano inferiori alla media nazionale in tutte le regioni del Mezzogiorno ad eccezione della Sardegna.

Nel 2014 è aumentata la produzione lorda elettrica da fonti rinnovabili e la sua incidenza sul consumo interno lordo di energia elettrica (37,3\% contro 33,7\% del 2013). Sul territorio, la produzione di energia elettrica da fonti rinnovabili si conferma in quantità nettamente superiore alla richiesta interna in Valle d'Aosta e nelle province autonome di Trento e Bolzano. Con una quota del $31,3 \%$ I'Italia si posiziona sopra la media Ue28 per consumi di energia elettrica generata da fonti rinnovabili $(25,4 \%$ nel 2013$)$.

\section{ECONOMIA E FINANZA PUBBLICA}

\section{Macroeconomia}

La quota dei consumi italiani sul Pil si attesta all' $80,6 \%$ nel 2014, mantenendosi più elevata rispetto alla media dei 28 paesi Ue $(77,7 \%)$ e a quelle dei principali paesi dell'area. L'incidenza degli investimenti è poco meno del $17 \%$, in questo caso inferiore alla media europea.

Tra il 2010 e il 2014 la produttività del lavoro italiana è aumentata solamente dello 0,3\%, un ritmo decisamente inferiore a quello medio europeo e dei principali paesi.

L'inflazione al consumo si è confermata prossima allo zero anche nel 2015. A livello territoriale sono risultate in deflazione metà delle regioni ma il rallentamento si è manifestato con maggiore intensità nel Mezzogiorno. In un quadro di diffusa attenuazione o azzeramento delle dinamiche dei prezzi, soltanto 14 paesi europei oltre al nostro registrano tassi positivi, sebbene contenuti e non superiori al punto percentuale.

Nel 2014 sono aumentate le quote di mercato dell'Italia sulle esportazioni mondiali di merci; nella graduatoria europea il nostro Paese è in quarta posizione. Dopo tre anni di calo, torna a crescere anche la presenza di operatori commerciali italiani nell'area Ue28 (oltre 153 mila). A livello territoriale, il contributo principale alle vendite italiane sui mercati esteri proviene dal Nord-ovest $(40,2 \%)$ e dal Nord-est $(31,8 \%)$; il Mezzogiorno registra una quota molto limitata $(10,2 \%)$ e in diminuzione per il secondo anno.

\section{Finanza pubblica}

La Pubblica amministrazione italiana nel 2014 spende circa 13,5 mila euro per abitante, un valore leggermente superiore a quello medio dell'Ue28. Tra le grandi economie dell'Unione, Francia, Germania e Regno Unito presentano livelli più elevati, mentre la Spagna spende meno dell'Italia. 